\title{
Lunar Regolith Simulant Feed System for a Hydrogen Reduction Reactor System
}

\author{
R. P. Mueller ${ }^{1}$ and I. I. Townsend $\mathrm{III}^{2}$ \\ ${ }^{1}$ Advanced Systems Division, M/S:KT-C, NASA, Kennedy Space \\ Center, Florida 32899, USA \\ ${ }^{2}$ Arctic Slope Research Corporation (ASRC), Kennedy Space \\ Center, Florida 32899, USA
}

\begin{abstract}
.
One of the goals of In-Situ Resource Utilization (ISRU) on the moon is to produce oxygen from the lunar regolith which is present in the form of Ilmenite (FeTiO3) and other compounds. A reliable and attainable method of extracting some of the oxygen from the lunar regolith is to use the hydrogen reduction process in a hot reactor to create water vapor which is then condensed and electrolyzed to obtain oxygen for use as a consumable. One challenge for a production system is to reliably acquire the regolith with an excavator hauler mobility platform and then introduce it into the reactor inlet tube which is raised from the surface and above the reactor itself. After the reaction, the hot regolith $(\sim 1000 \mathrm{C})$ must be expelled from the reactor for disposal by the excavator hauler mobility system. In addition, the reactor regolith inlet and outlet tubes must be sealed by valves during the reaction in order to allow collection of the water vapor by the chemical processing sub-system. These valves must be able to handle abrasive regolith passing through them as well as the heat conduction from the hot reactor. In 2008, NASA has designed and field tested a hydrogen reduction system called ROxygen in order to demonstrate the feasibility of extracting oxygen from lunar regolith. The field test was performed with volcanic ash known as Tephra on Mauna Kea volcano on the Big Island of Hawai' $i$. The tephra has similar properties to lunar regolith, so that it is regarded as a good simulant for the hydrogen reduction process. This paper will discuss the design, fabrication, operation, test results and lessons learned with the ROxygen regolith feed system as tested on Mauna Kea in November 2008.
\end{abstract}

Keywords: Lunar Outpost, Lunar Regolith, Regolith Feed System, Tephra , In-Situ Resource Utilization (ISRU), Hydrogen Reduction, Mauna Kea, Hawai'i, Simulant.

\section{INTRODUCTION}

The production of oxygen from regolith on the moon is an attractive technology option, since it can reduce the total mass of consumables that must be taken to the moon from the Earth. If the mass of oxygen produced and consumed on the moon significantly exceeds the mass of the equipment that is transported from Earth to the moon in order to make this possible then there will be a benefit to the overall architecture. A mission planner has additional variables that must be included in the decision trade-offs which include reliability, cost, safety, maintainability and operability. That is why it is necessary to go beyond the fundamental chemical processes of oxygen production from regolith in a lab environment and show that it is a viable technology by demonstrating prototypical hardware in field environments where the unexpected often happens and thus provides a more realistic test of the hardware and all of the related aspects of oxygen production operations.

The National Aeronautics \& Space Administration (NASA) In-Situ Resource Utilization (ISRU) project in the Exploration Technology Development Program (ETDP) has designed and built an oxygen production device prototype called ROxygen. This first generation prototype was tested in November 2008 on Mauna Kea volcano in the Big Island of Hawai' $i$. The ROxygen system is a self contained hydrogen reduction reactor payload that is part of the modular Outpost Precursor Testbed for ISRU \& Modular Architecture (OPTIMA) system. The goals of the OPTIMA test were to: 
- Demonstrate excavation and regolith delivery to/from ISRU plant

- Demonstrate oxygen extraction from regolith at outpost production rate

- Demonstrate oxygen storage

- Demonstrate system integration, modularity of modules for swapping, and surface operations
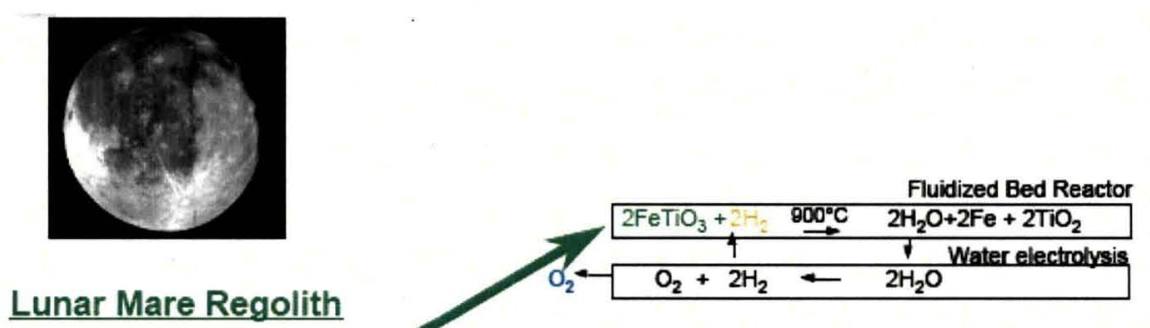

Hydrogen Reduction of Ilmenite/glass

\section{Lunar Mare Regolith} Process
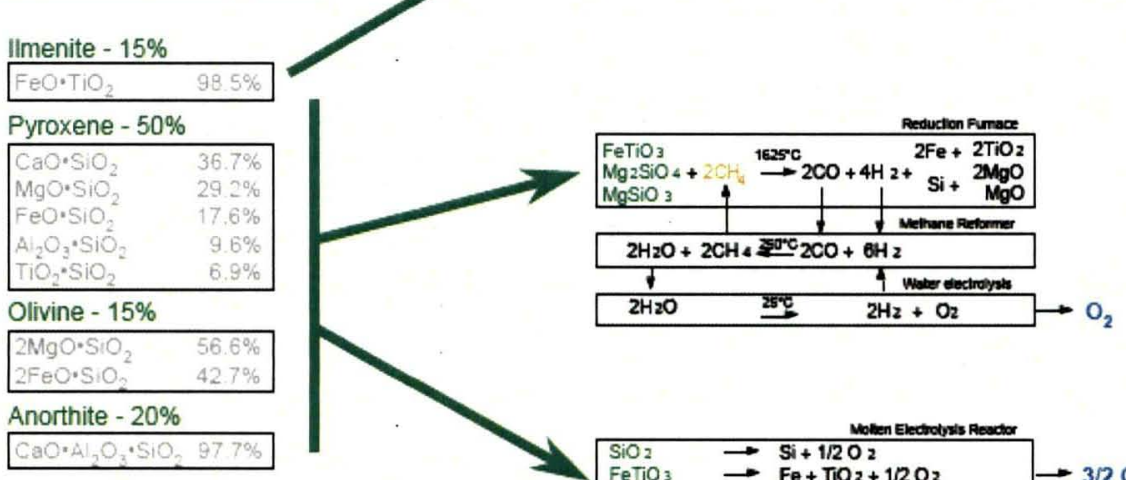

Methane Reduction (Carbothermal)

Process
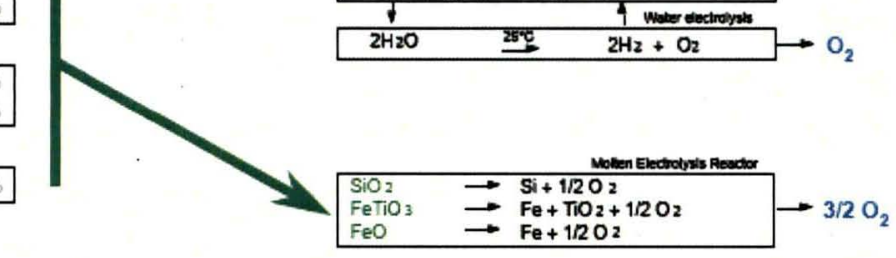

Molten

Electrolysis

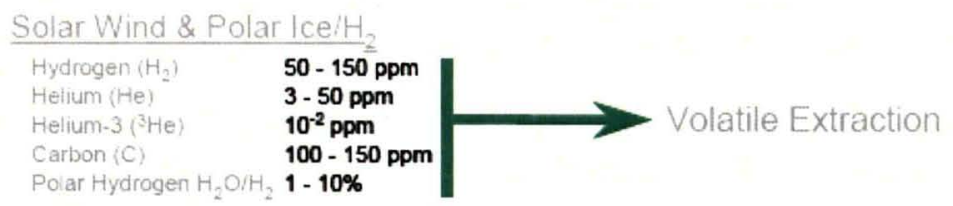

Figure 1: Methods of Lunar Oxygen Production under Consideration by NASA

The NASA OPTIMA modules consisted of:

1. Mobility, Excavation, and Beneficiation Module

2. H2 Reduction Reactor Module (ROxygen)

3. Carbothermal Reduction Reactor Module

4. Water Processing Module

5. Storage Module

This paper will deal only with item 2: H2 Reduction Reactor module as demonstrated by the NASA built ROxygen system. The NASA ROxygen fluidized bed and auger hydrogen reduction reactor makes oxygen at approximately $660 \mathrm{~kg} / \mathrm{yr}$ which is about $2 / 3$ of the scale required for the initial stages of a Lunar Outpost being designed for the NASA architecture ( $1000 \mathrm{~kg} /$ year). It consists of a cylindrical reactor that has an internal auger system that stirs the regolith to enhance heat transfer and prevent regolith sintering. Furthermore this paper will be dedicated to reporting on the development of a sub-system of the ROxygen system: the lunar regolith simulant feed system.

The basic design criteria for the ROxygen regolith simulant feed system were to deliver regolith simulant from the ground level to the inlet tube of the ROxygen reactor cylinder which was constrained to being in a vertical configuration to enable a gravity feed of regolith simulant into and out of the reactor cylinder. In addition, the 
regolith simulant feed system had to channel the spent regolith from the outlet tube of the reactor to a regolith disposal location on the ground for eventual pickup by a disposal hauler machine. The feed system had to be simple, lightweight, reliable, safe and capable of transportation and field assembly. The packaging of the feed system into the overall ROxygen prototype envelope was a major driver in the generation and selection of various concepts. In order to maintain pressure inside the reactor, the regolith feed system had to be capable of isolating the reactor at the inlet and outlet tubes. Therefore regolith tolerant valves were researched and developed, which had seals that are capable of maintaining a desired pressure even after cold and hot regolith had passed through them.

Since the energy requirements of a reactor can be quite large, as it heats the regolith to a temperature approaching 1000 degrees Celsius, it was highly desirable to incorporate a heat recuperation system into the regolith feed system. The hot regolith that was ejected from the reactor had to heat the cold regolith in the input hopper prior to introducing it into the reactor for the next batch of oxygen extraction.

The regolith simulant that was used for the ROxygen reactor is the local volcanic ash that can be found on Mauna Kea volcano, on the big Island of Hawai'i. This volcanic ash is known as "Tephra". The tephra was obtained locally and dried and sieved to a particle size of $1 \mathrm{~mm}$ or less prior and placed in an excavation area. The tephra was then excavated and transported to the regolith feed system inlet hopper by a small mobile NASA excavation machine called CRATOS.

\section{CONCEPTS}

Many concepts were considered during the initial brainstorming phase of the regolith feed system design. Over twenty system concepts were generated and evaluated. The basic elements of the system and related methods were:

Reactor Chamber Shape:

- V-Shaped Bottom

- Cylindrical

- Angled-X

- Conical (Distributor Plate)

- Rectangular

Regolith Particle Feeding:

- Gravity Feed

- Screw Conveyor Feed

- Gas Feed/Pulse Flow

- Vibration

- Rotary Feed

Fluidization / Prevent Sintering:

- Crusher

- Flow Rate

- Centrifugal Flow

- Circulatory Flow

- Mechanical Vibration

- Mixers/Fins

Processing:

- Batch processing

- Continuous Processing 
Heating:

- Electrical Resistance

- Ceramic Electrical Resistance

- Induction

- Dielectric

- Infrared

- Impedence

Heat Recuperation:

- Solid-Solid Heat Transfer

- Solid-Gas Heat Transfer

- Heat Pipes

Regolith Withdrawal:

- Gravity Withdrawal

- Screw Withdrawal/Conveyor

- Gas withdrawal / Pulse Flow

- Vibration

- Rotary Feed

The combinations of the various concepts resulted in a very large number of possibilities. By narrowing these concepts down to viable systems with commercially available components, it was determined that there were about 6-10 concepts that may have been practical and within budget and schedule constraints. However the main considerations all revolved around the heater shape and configuration, heating type, batch processing and heat recuperation method. The feed system was determined to be a secondary system that had to comply with the optimized configuration of the reactor which was consistent with the goal of efficient oxygen production. However this resulted in additional constraints on the regolith feed system, which in turn determined the design.

The reactor design that was chosen from the options listed above was the cylindrical reactor with a smaller diameter resistance core heater in the central axis of the reactor cylinder. The top of the cylinder had a 1 inch inner diameter inlet tube and the bottom of the reactor had a 1 inch inner diameter outlet tube. These tubes dictated the primary interfaces for the regolith feed system.

The other primary interfaces were the interfaces between the mobile regolith excavator / hauler (CRATOS) on the inlet and outlet side of the regolith transfer system. It was determined that CRATOS could excavate, haul and dump a $10 \mathrm{~kg}$ payload of tephra to an inlet hopper by climbing up a ramp inclined at about 15 degrees to the horizontal surface. On the outlet interface side, the hot tephra was dumped onto the surface for cooling, then the excavator hauler came back and disposed of it.

\section{REGOLITH FEED SYSTEM PHASE I PROTOTYPE}

In order to meet the constraints that were imposed on the regolith feed system by the reactor design, and still meet all the design criteria, the method that was ultimately chosen following an evaluation process of all available concepts, was the use of a combined gravity feed and auger feed system. The gravity feed was used to load the input hopper, and feed the tephra into the auger feed system. The auger feed system was necessary in order to raise the regolith simulant (tephra) against the force of gravity to the top of the reactor which was approximately 5.5 Feet off the ground. Once the regolith had been sufficiently raised above the reactor ( 7 Feet) it was possible to use gravity feed again in order to flow the regolith into the reactor through an inlet valve. After passing through the inlet valve and through the inlet tube, the tephra was introduced into the reactor. 
The goal of heat recuperation was achieved by devising a "hopper within a hopper" system. The hot regolith can be fed into the inner concentric hopper where it transfers heat to the outer hopper which is filled with cold regolith. The warmed input regolith is then pre-heated which results in lower heating energy requirements for the reactor. This system was prototyped and proven to flow in $1 \mathrm{G}$ and $1 / 6^{\text {th }} \mathrm{G}$ (Reduced Gravity Flight). It was shown to be viable, but due to budget, schedule and system integration issues it was determined that the heat recuperation would be implemented in a phase II design, which will be built and tested in the future.
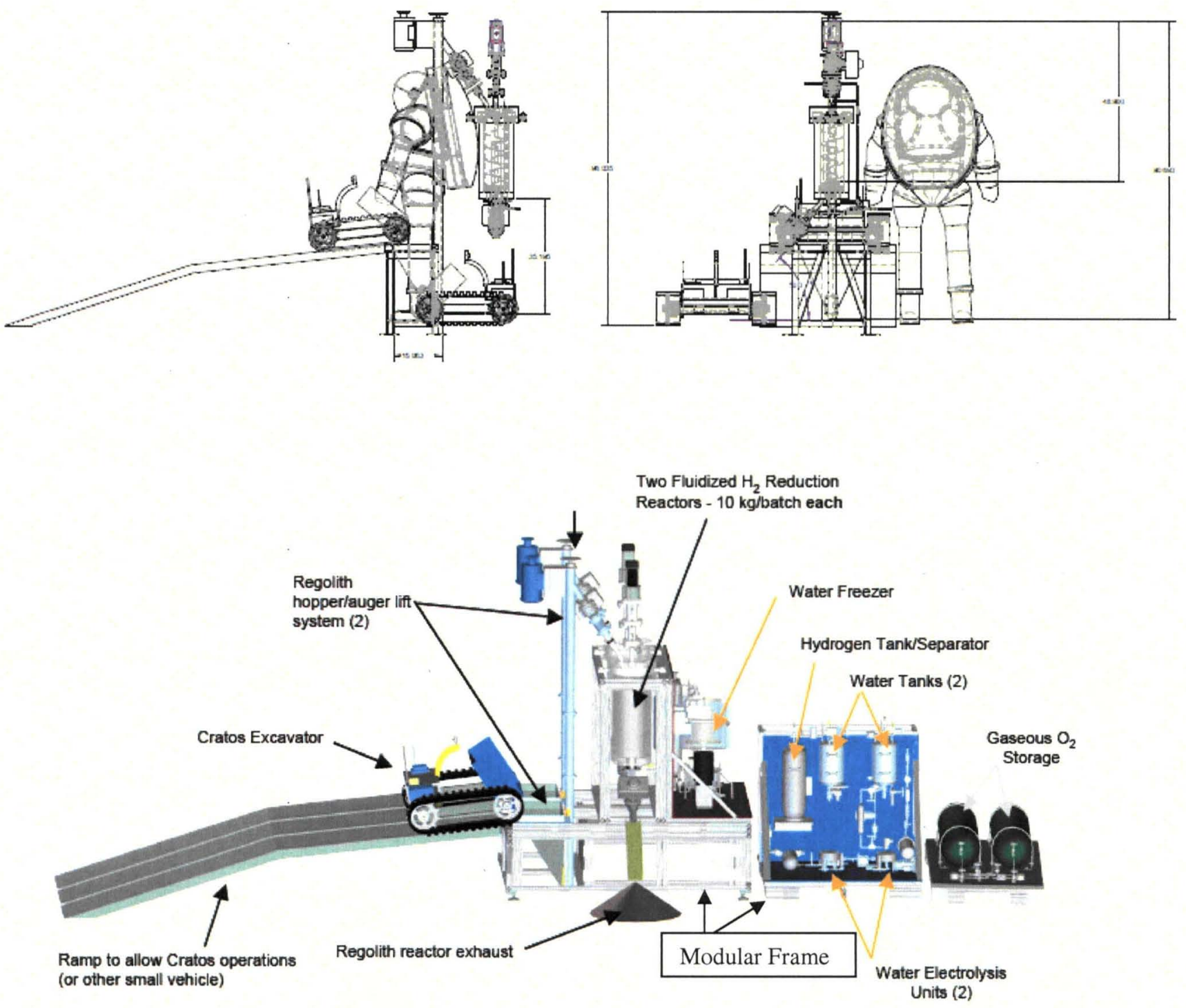

NASA ROxygen $\mathrm{H}_{2}$ Reduction System

Figure 2: Final Configuration of Phase I ROxygen Prototype

The final configuration of the ROxygen system is shown in Figure 1 above. A modular frame supplied by 80/20 inc. was assembled to provide structural support for the components. The benefits of using this modular frame were short lead times, quick and easy assembly, re-configurability and field assembly feasibility. Two reactors were hung 
from this frame in order to provide a redundant system that is capable of parallel processing; while one reactor is heating the tephra and extracting. $\mathrm{H} 2 \mathrm{O}$, then the other reactor system can pre-heat and feed the tephra in to the reactor. This meant that two regolith feed systems were provided under the same philosophy of redundancy and parallel processing. The regolith feed system was loaded with an input hopper shown at the end of the ramp. A steel tube, encapsulated a steel auger element that was connected to a motor mounted at the top of the steel tube. The tephra was transported by the flights of the auger inside the tube until an opening was reached at the top of the tube. At this point the tephra was gravity fed to the reactor inlet tube.

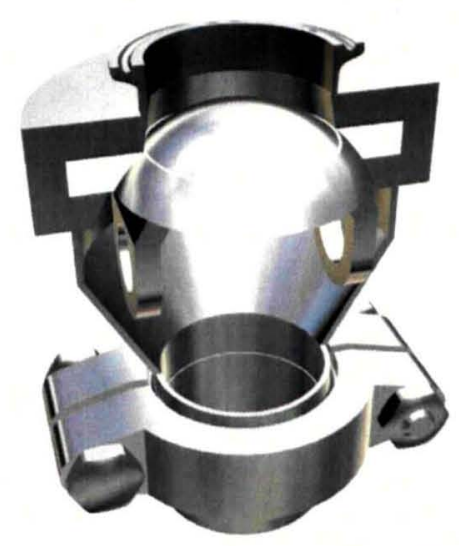

Figure 3: Gemco, inc Spherical Disc Valve

The reactor was sealed by using 2 inch diameter spherical disc valves with metallic seals that were provided by Gemco, inc. These valves were jointly developed by NASA and Gemco, in order to provide abrasion resistance and seal dust tolerance from the regolith simulant as it passed through the valves. The valves also had to be able to withstand the 1000 Degree Celsius tephra as it flowed through the outlet side. Finally, the valves had to be leak tight during the batch reaction process, even after multiple batches of hot regolith had passed through them.

\section{TESTING ON MAUNA KEA, HAWAI'I}

From November 6-16, 2008, NASA held an ISRU field test on Mauna Kea, Hawai'i in a location near the visitor's center which is called "Wahine" Valley. The valley had the advantage that over time, fine grained tephra had been washed into the valley basin, and there is sparse vegetation with a low organic content to the tephra. In addition, transportation required good packaging of all hardware, similar to the requirements of space transportation and the environment was extremely dusty which served to provide additional operational challenges.

The ROxygen hardware was assembled on site and checked out. The testing showed that $10 \mathrm{~kg}$ of tephra could be reliably transferred into the reactor in 470 seconds. Eventually it was determined that $8 \mathrm{~kg}$ was the optimal reaction mass and this mass of tephra was transferred in 408 seconds with smooth flow out of the hopper and into the auger lift mechanism. The tephra was also successfully withdrawn from the reactor with gravity feed through the outlet valve. The valves performed well but required adjustment in order to achieve the desired leak rate without having excessive torque on the actuation mechanism.

Multiple reactor operations with Reactor Module were performed. The goal was to process three batches with one of the two reactors performing back to back tests. These tests were successfully performed and it was shown that water could indeed be made from tephra and subsequently electrolyzed to produce oxygen.

Severe dust storms and fluctuating temperatures added to the challenges of the field test and emphasized the fact that the equipment design must be very robust to survive the environmental conditions it will face on the moon which will be much more severe than anything that can be experienced in a field test on Earth. 


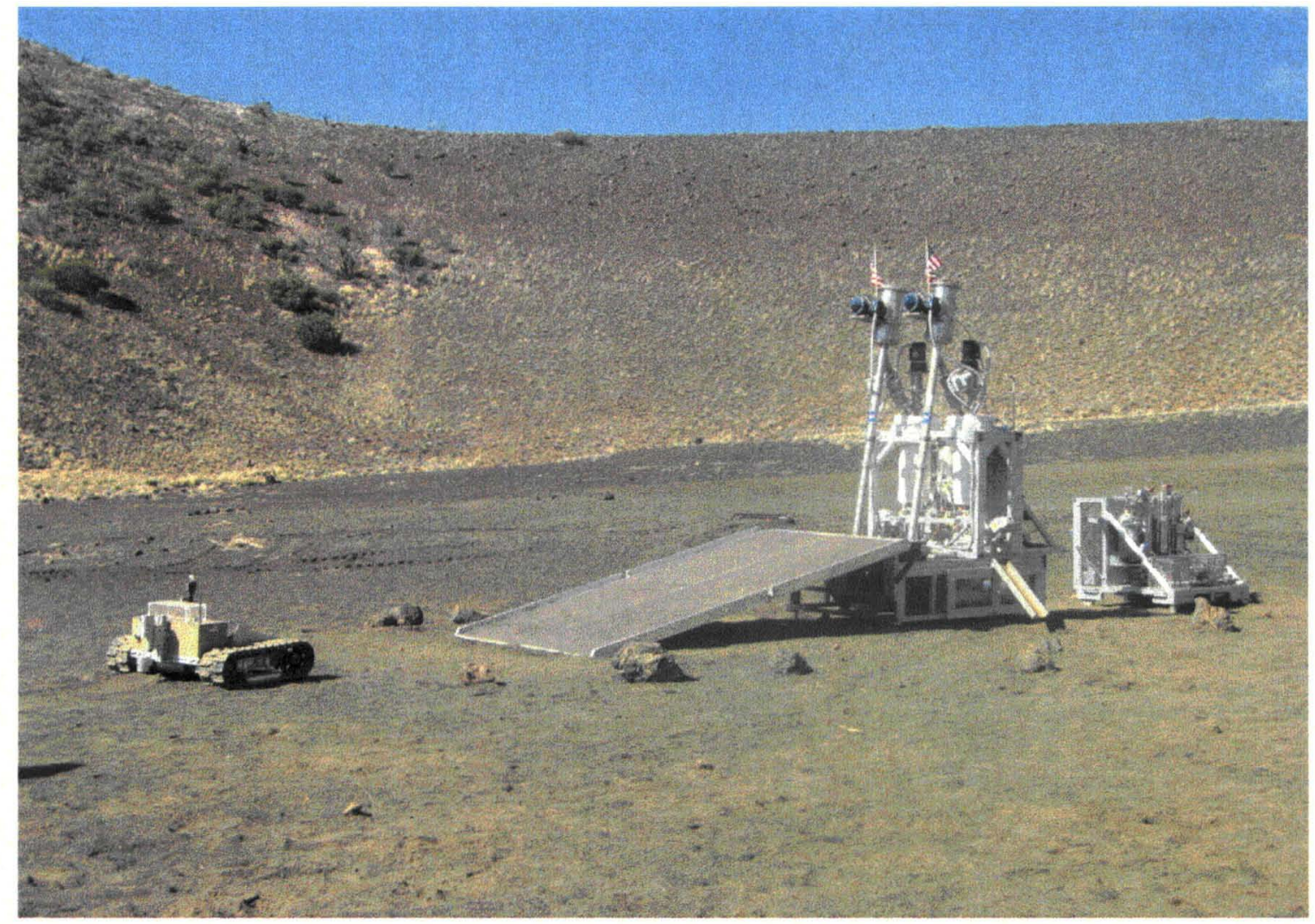

Figure 4: ROxygen System on Site in Wahine Valley, Mauna Kea, Hawai'i

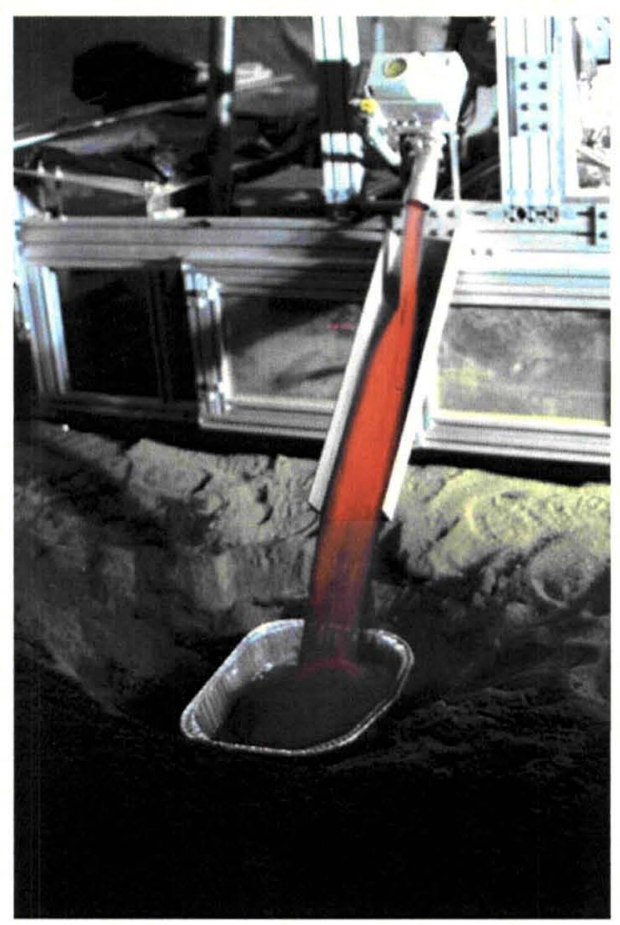

Figure 4: Hot Tephra flowing out of the ROxygen Reactor 


\section{LESSONS LEARNED}

There were several lessons learned during the design phase, assembly and integration phase of the ROxygen system, and also during the field testing on Mauna Kea.

The design team was geographically dispersed between Florida, Texas, Ohio, California and New Jersey. Collaborative work was required using Computer Aided Design (CAD) tools and electronic data exchange as well as more traditional methods such as team telecons and white board brainstorming and design sessions. Communication by having weekly telecons with frequent $\mathrm{CAD}$ file exchange was vital to making this team function effectively.

Lead times for regolith tolerant valves with metal seals were a challenging aspect of this design. Regolith valves are highly specialized and few vendors were capable or willing to tackle this challenge. Lead times can be as long as 68 months for a custom valve design. The motors on top of the auger tubes were not aesthetically pleasing and increased the volumetric envelope of the ROxygen system substantially. A new design concept has evolved which can eliminate these motors on top of the tubes and this may be implemented in Phase II. Other designs have also been developed during the Phase I concept generation, and another promising system involves pneumatic conveying of regolith.

During the assembly and integration phase the input hopper system was built in a mock up version with lexan for flow visualization. The flow was immediately visible and verifiable. Many hours of analysis were saved by rapid prototyping and experimental testing methods. In addition an opportunistic reduced gravity flight on the C-9 NASA aircraft at Ellington field proved that the concept would work in $1 / 6^{\text {th }} \mathrm{G}$ with gravity feed albeit with some enhancements to accelerate flow.

It was also discovered that the auger worked well with JSC-1a in the vertical configuration but was inconsistent with tephra in that orientation. As a result, it was found during the checkout stage that the auger system worked reliably with tephra when inclined at an angle of 10 degrees from the vertical. The auger system is sensitive to particle size and type. Large pebbles tended to jam the auger, so that a size sorting system is needed. This was achieved with pre-sieving and final sieving with a mesh screen mounted on the auger opening.

\section{CONCLUSION}

The OPTIMA tests on Mauna Kea, Hawai'i in November of 2008 clearly demonstrated the feasibility of producing oxygen at rates equivalent to $660 \mathrm{~kg} /$ year in a Lunar Outpost. The regolith feed system performed well and met all objectives. Batches of $8 \mathrm{~kg}$ of tephra were consistently, repeatably and successfully fed from the input hopper to the reactor and then expelled to the surface. High temperatures and abrasive particles were handled well with this system. Lifetime testing is required to determine wear rates and ultimate system reliability. The system proved to be very robust in limited field testing. Phase II of the ROxygen system design will incorporate the lessons learned and increase the fidelity of the prototype hardware.

\section{REFERENCES}

Caruso, J.J., Greer, Lawrence C.; John, Wentworth T.; Spina, Dan C.; Krasowski, Mike J.; Abel, Phillip B.; Prokop, Norman F.; Flatico, Joseph M.; Sacksteder, Kurt R.,"Cratos: A Simple Low Power Excavation and Hauling System for Lunar Oxygen Production and General Excavation Tasks," PTMSS Conference, Sudbury, Ontario, Canada, 1013 Jun. 2007

Cooke, D. et al, Exploration Strategy and Architecture, Implementing the Vision, $2^{\text {nd }}$ Space Exploration Conference, AIAA, Houston (2006).

Sanders, G.; Larson, W., "NASA In-Situ Resource Utilization (ISRU) Development \& Field Testing," Presentation to "Hawai'i's Aerospace Industry: The Next Frontier", Honolulu, The Hawai'i State Capital Auditorium, August 21,2008 